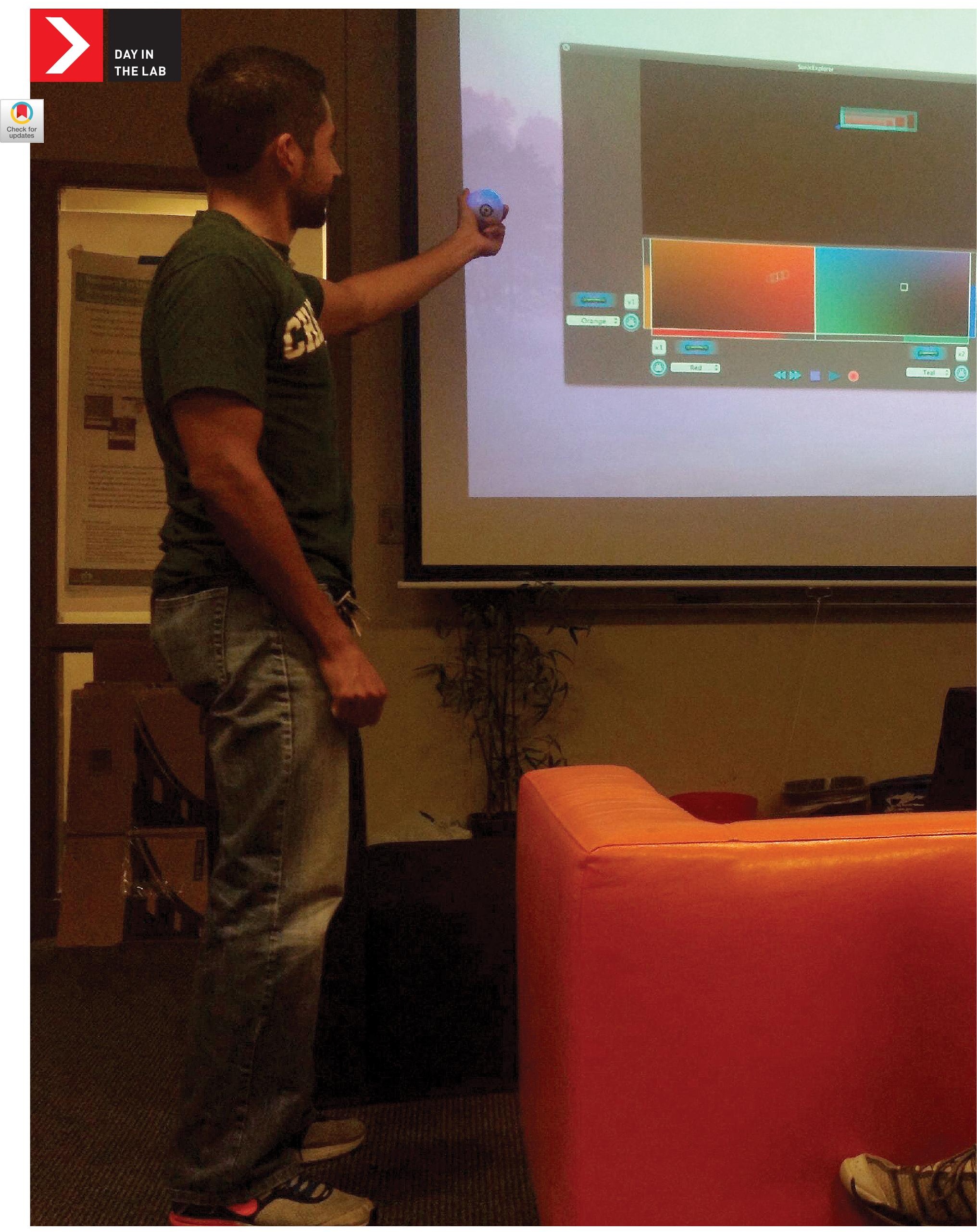




\section{UNC Charlotte HCI/InDe Lab}

As told by Celine Latulipe, Heather Lipford, Mary Lou Maher, and David Wilson
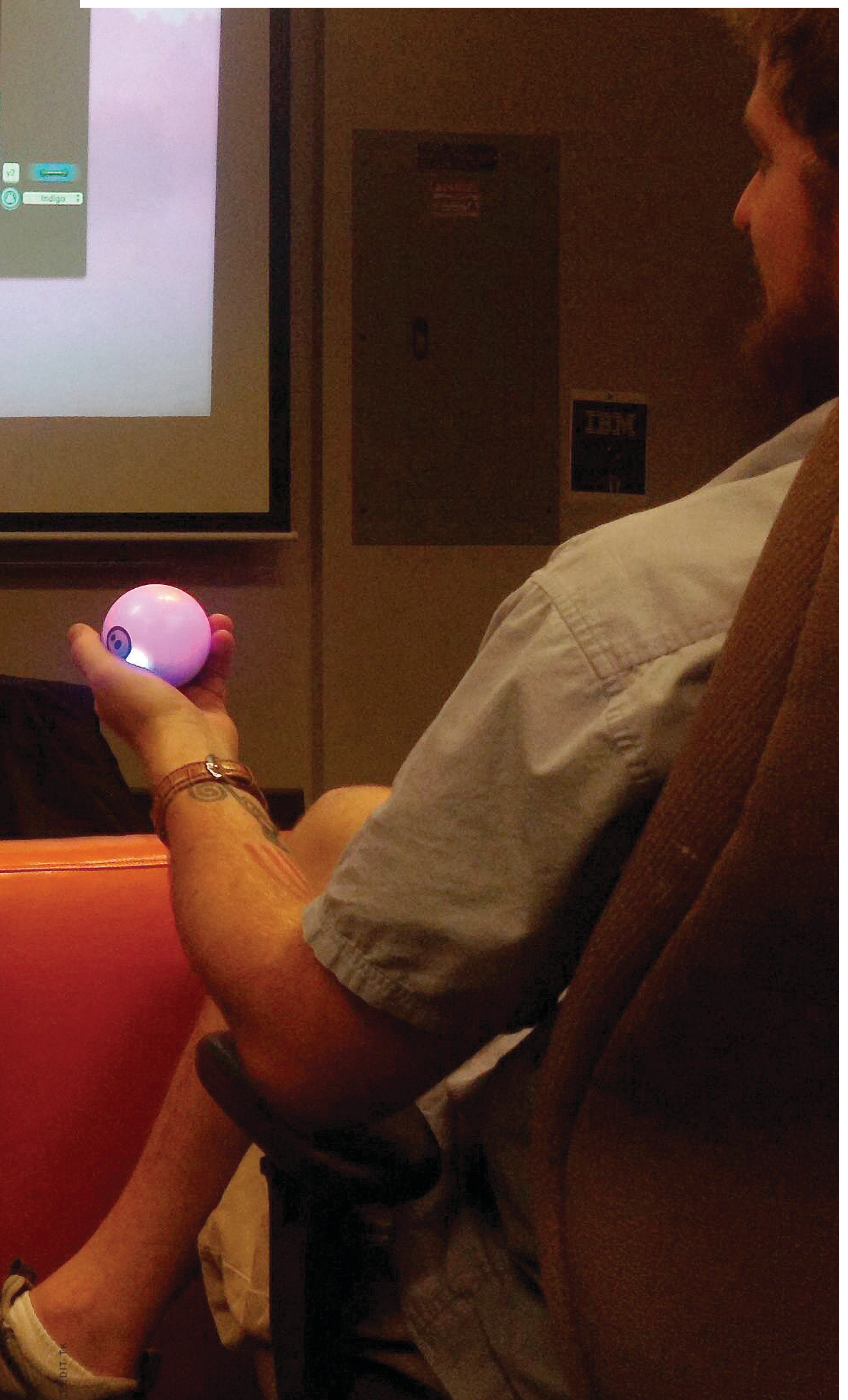

How many people are in the lab, and what is the mix of backgrounds and roles? Four faculty members share the lab space. In a typical semester, our lab spaces house about 10 to 12 doctoral students and one or two postdocs; sometimes a special undergrad will also be given space. The graduate students who work with us come from computing, architecture, and psychology. We have a joint master's program between computing and architecture; those students also play an important role in bringing design expertise to research projects in the lab.

Briefly describe a day in the life of your lab. It's hard to describe a typical day 

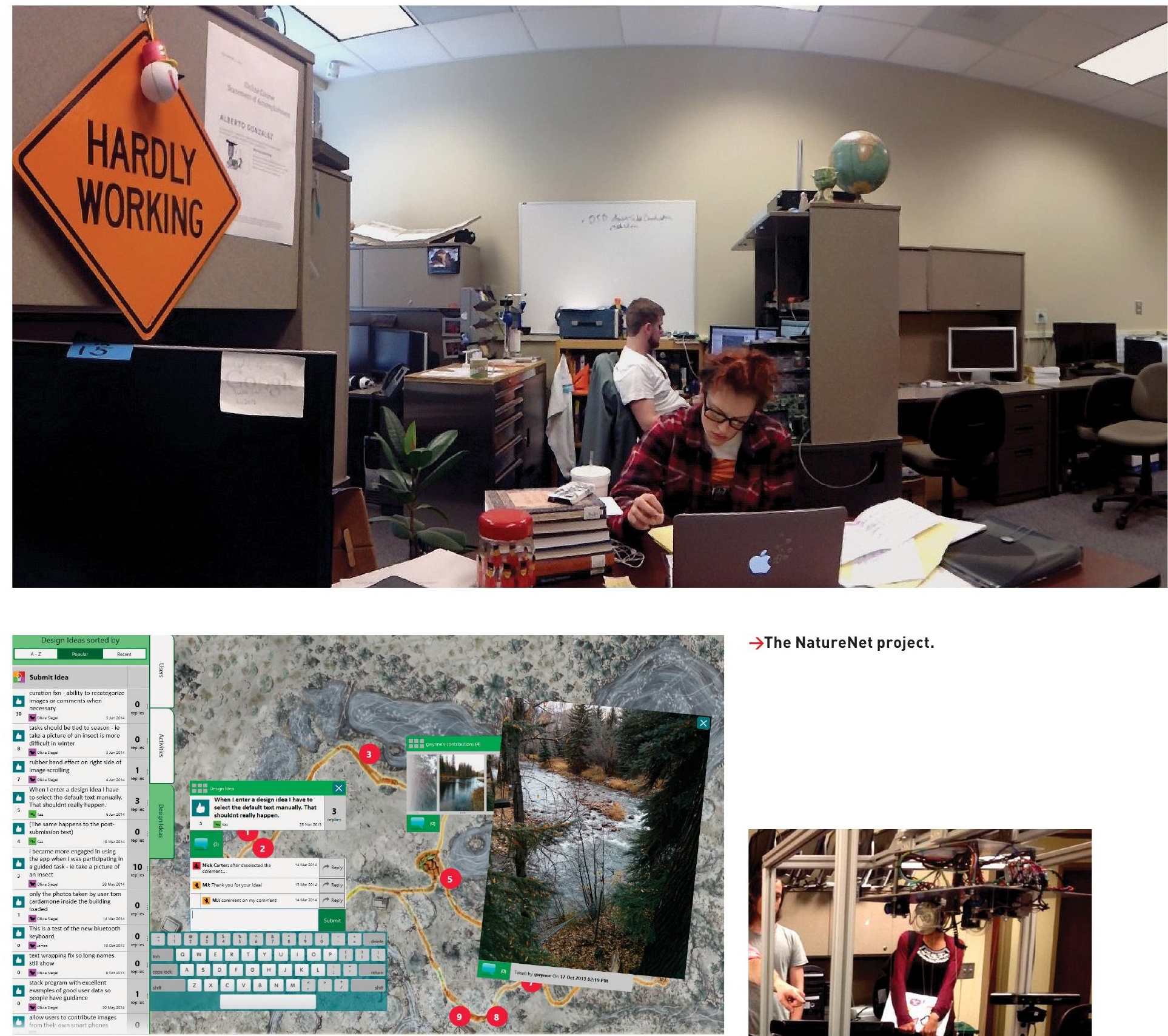

$\rightarrow$ The NatureNet project.

Long ago, an undergrad student wanted to create a program that would allow him to paint with the sound of his whistle. It was a bit crazy, but we gave him the space to do it.

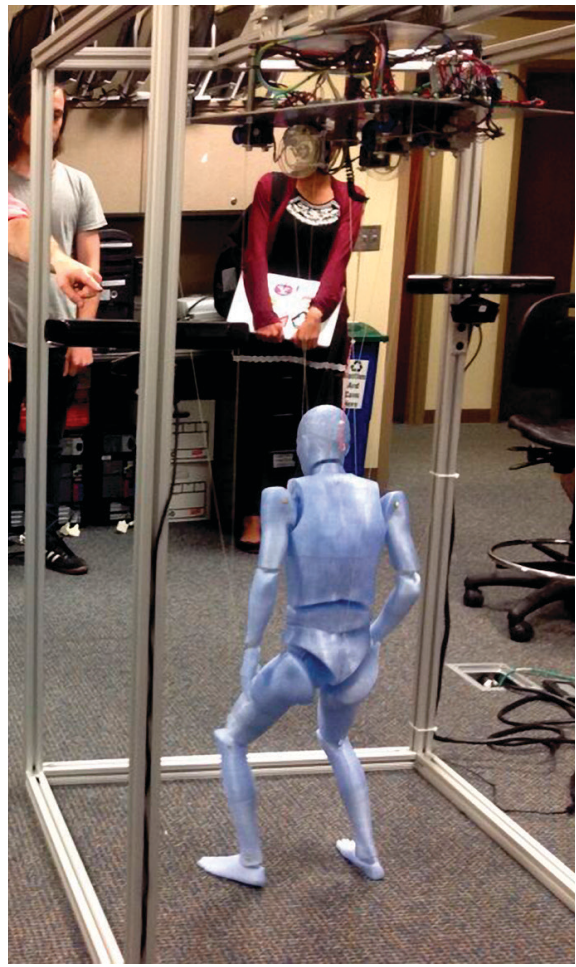



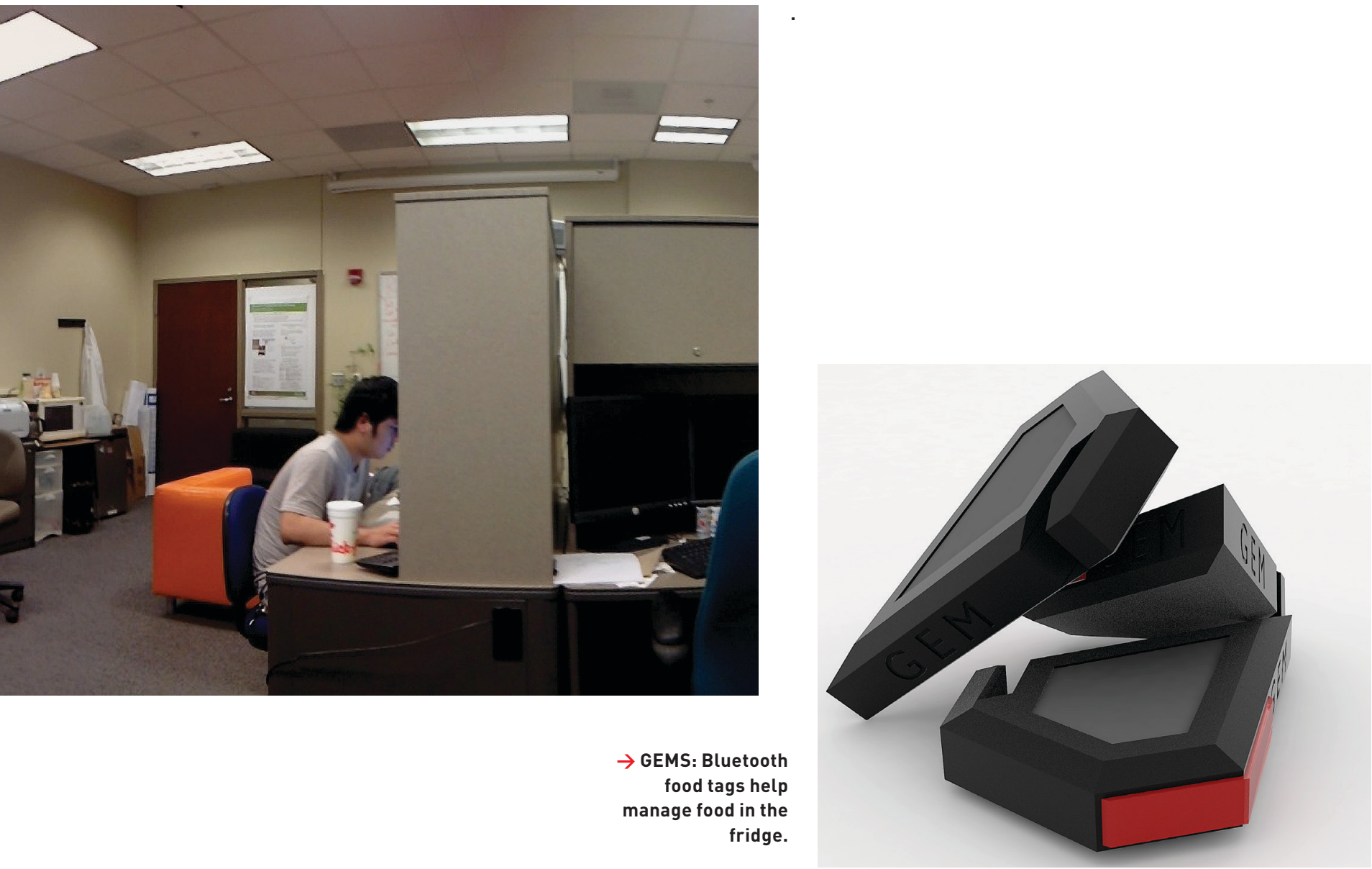

in our lab, as the variety of research is quite broad. Starting with the Dance.

Draw project, there has been significant emphasis on IT support for creativity and the arts. This has led to a focus on video-centered collaboration and computing education research. We also have faculty and students focusing on usable security and privacy, interfaces for secure programming, recommender systems, and tangible interaction.

Because of the wide variety of research, it's typical to find people working furiously toward paper deadlines. While the research projects in our lab are quite varied, our students consistently help each other by giving feedback on research papers, study designs, and new interfaces; assisting with user study recruitment; and anything else that is needed. It's a very supportive environment.

What is one feature of your lab that you could not do without? The interactive touch table in the InDe Lab is used for the NatureNet project and for other student projects. This table gives students the ability to dive into the design of touch and gestural interaction. The NatureNet project brings a sustainability and ecology perspective into the lab, and connects students and faculty at UNC Charlotte with collaborators at other universities and at national parks.

\section{What is one feature of your lab you} want and do not have? While we do have some 3D printers (Makerbots and a Cube), we do not have a full fabrication setup with a 3D scanner, a vacuum sublimation heat-press, a laser cutter, and other state-of-the-art fabrication equipment. Given the range of application areas, from medical devices and clothing to sustainable building materials, we feel that having more of this type of equipment would help us show students how creative and purposeful computing research can be.
What is the one thing you see as most important about the work you do there? Encouraging creativity and playfulness is something we think is very important. Not every piece of equipment is purchased for a specific purpose. Sometimes we just want students to experiment and play with new technology. We find this helps spur new ideas and creates an environment where experimentation and creativity are valued. Long ago, an undergrad student wanted to create a program that would allow him to paint with the sound of his whistle. It was a bit crazy, but we gave him the space to do it and that algorithm ended up being used for visualizations in an interactive dance and in a project to help support seniors aging in place. While user-centered design to solve problems is a large part of what we do, sometimes it's also great to be inspired by fun new technology or algorithms. 